\title{
Evolution of photoperiod sensing in plants and algae
}

\author{
Gloria Serrano-Bueno ${ }^{1}$, Francisco J Romero-Campero ${ }^{1}$, Eva I Lucas-Reina ${ }^{1+}$, Jose M Romero \\ and Federico Valverde*
}

Plant Development Unit. Institute for Plan Biochemistry and Photosynthesis, CSIC-Universidad de Sevilla. 49th , Americo Vespucio Av. 41092-Sevilla. SPAIN. + Present address: IHSM-UMA-CSIC, Departamento de Biología Molecular y Bioquímica, Universidad de Málaga, 29071 Málaga, Spain.

\begin{abstract}
Measuring day length confers a strong fitness improvement to photosynthetic organisms as it allows them to anticipate light phases and take the best decisions preceding diurnal transitions. In close association with signals from the circadian clock and the photoreceptors, photoperiodic sensing constitutes also a precise way to determine the passing of the seasons and to take annual decisions such as the best time to flower or the beginning of dormancy. Photoperiodic sensing in photosynthetic organisms is ancient and two major stages in its evolution could be identified, the cyanobacterial time sensing and the evolutionary tool kit that arose in green algae and developed into the photoperiodic system of modern plants. The most recent discoveries about the evolution of the perception of light, measurement of day length and relationship with the circadian clock along the evolution of the eukaryotic green lineage will be discussed in this review.
\end{abstract}

\footnotetext{
*Corresponding author: federico.valverde@ibvf.csic.es

${ }^{1}$ Contributed equally to this work.
} 


\section{Introduction}

Earth rotation around its axis and around the sun produces predictable day length (photoperiod) changes through the seasons that plants use, via sophisticated mechanisms, to measure time and take crucial physiological decisions [1]. Photoperiodism, or the ability to detect day length, is present in early photosynthetic eukaryotes so that algae can produce several photoperiod responses [2]. This way, during the green lineage evolution, photoperiodism pervaded into the major physiological systems, allowing them to predict the passing of the seasons and prepare plants for year-round predictable changing conditions.

The photoperiod sensing system involves a way to detect light (photoreceptors) and an internal system to measure time (circadian clock). In time they became so important for unicellular free living algae that for some marine picoeukaryotes $90 \%$ of its transcriptome is controlled by the clock [3]. However, more evolved and flexible species like modern plants, which developed the capacity to adapt to different environments, have reduced this number to less than 50\% [4]. Paradoxically, more intertwined, complex systems allowed for a more independent response to external cues, thus permitting the colonization of ever demanding new niches and the acquisition of novel and complex physiological functions [5].

\section{Photoreceptors evolution}

Living organisms use a cluster of photoreceptors to measure the quality, quantity and direction of light to modulate physiological responses to changing lights [6]. This is particularly important for photosynthetic organisms that require light energy for photosynthesis and consequently to grow and develop. Photoreceptors can be divided into three groups according to the light quality they detect. Red and far-red lights are absorbed by phytochromes (PHYs) while three types of photoreceptors perceive the blue/UV-A: Cryptochromes (CRYs), Phototropins (PHOTs) and three plant-specific LOV/F-box/Kelch-repeat proteins ZEITLUPE (ZTL), FLAVIN-BINDING KELCH REPEAT FBOX (FKF), and LOV KELCH REPEAT PROTEIN 2 (LKP2). Finally, UV RESISTANCE LOCUS 8 (UVR8) was recently shown to be a UV-B photoreceptor [7]. Excellent reviews on plant and algae photoreceptor structure and function have recently been published [8-13].

The specific photoreceptor set has evolved across photosynthetic eukaryotes (Figure 1). In the chlorophyte model alga Chlamydomonas reinhardtii, UV-B light is detected by UVR8 while blue/UV-A is detected by one PHOT (pho1), two DASH (Drosophila, Arabidopsis, Synechocystis and Human) CRYs, one plant-like CRY (pCRY) and one animal-like CRY (aCRY). The latter can respond both to blue and red light [12,14-15]. In the fern Adiantum capillus-veneris four canonical plant PHYs have been identified, two PHOTs and five CRYs [16]. Ferns include a specific neochrome, a chimeric photoreceptor consisting of an $\mathrm{N}$-terminus $\mathrm{PHY}$ domain and several $\mathrm{C}$ terminus PHOT domains that can sense both blue and red/far-red light to regulate 
chloroplast movement and phototropism [6]. However, UV-B photoreceptors have not been described in ferns, their absence justified by their growth habits under low-light angiosperm canopies. In the model plant Arabidopsis thaliana, red/far-red lights are detected by five PHYs (A-E), blue/UV-A by two PHOTs, three CRYs, and ZTL/FKF1/LKP2 proteins [6,11,13], while UV-B by a canonical UVR8 (Figure 1A). Increase in photoreceptors number and function during plant evolution has been related to fitness improvement [9].

In plants and algae, photoperiod regulates a number of processes including photomorphogenesis, growth, flowering, stress tolerance and circadian rhythms [1618]. In darkness, some of these pathways are inhibited by CONSTITUTIVE PHOTOMORPHOGENIC 1 (COP1), a RING-finger E3 ubiquitin ligase. During the day, COP1 is inhibited by the photoreceptors allowing the activation of photoperiodicdependent processes (Figure 1A). At night, COP1 interaction with SUPPRESSOR OF PHYTOCHROME A (SPA1) targets the transcription factors ELONGATED HYPOCOTYL 5 (HY5) and CONSTANS (CO) for ubiquitination and degradation, suppressing photomorphogenesis and flowering respectively [20,21]. Photoactivated CRY1, CRY2 and PHYA directly bind SPA1 and inhibit the formation of COP1-SPA1 complex [19]. PHYB also promotes COP1-SPA1 dissociation and photomorphogenic development [22]. COP1 interaction with EARLY FLOWERING 3 (ELF3) induces degradation of GIGANTEA (GI), a circadian clock associated protein, process inhibited by CRY1/CRY2 in blue light [23]. Upon UV-B irradiation, UVR8 monomerizes and interacts with COP1, promoting the expression of HY5, which is responsible for activation of UV-B responsive genes [24]. In Chlamydomonas cells grown under blue light, CrCO (Chlamydomonas CO homolog [18]) transcript levels are lowered in the crcrya mutant [15], suggesting a possible CrCO activation by CrCRYa. Besides, UV-B perception and signalling in Chlamydomonas is mediated by CrUVR8 that interacts with CrCOP1, although CrHY5 implication has not been demonstrated [14]. RING-finger E3 ligases homologues to COP1 can be also found in other microalgae. Therefore, it seems that a central role for COP1-like signalling mechanisms was already established in chlorophytes and evolved to the complexity found in modern plants.

\section{Circadian clocks in algae and plants}

Circadian clocks are molecular mechanisms that generate rhythmic or oscillating signals with a period of approximately 24 hours. They are ubiquitous systems present in almost all eukaryotic organisms, but in spite of the long evolutionary distance among them, they are composed of strikingly similar gene networks comprising intertwined positive and negative feedback loops [25]. These genes are not always orthologues, suggesting a convergent and independent evolutionary history. Recently, high throughput sequencing and genome-wide phylogenetics are starting to unveil an 
interesting evolution of the gene network underpinning the circadian clock composition in the green lineage.

Three interlocked feedback loops have been identified in the model species Arabidopsis; morning, central and evening loops (Figure 2A) [26]. The key gene in the morning loop is CIRCADIAN CLOCK-ASSOCIATED 1 (CCA1) that codes for a MYB transcription factor [27] with a conserved N-terminal SHAQKYF motif (Figure 2B). MYB family has gone through an intense process of amplification and functional diversification in the green lineage [28] and CCA1 homologues are present in every plant taxa, from the single copy gene OtCCA1 in Ostreococcus [29] to the large gene family in Arabidopsis including CCA1, LATE ELONGATED HYPOCOTYL (LHY) and REVEILLE (RVE) 4, 6 and 8 [30]. All these homologues, with the exception of Chlamydomonas, exhibit the same expression pattern as Arabidopsis CCA1: A peak at dawn and a trough at dusk (Figure 2C).

ChIP-seq data has shown that CCA1 binds directly to the promoters of the other key genes in the morning loop, the PSEUDO-RESPONSE REGULATOR 9,7 and 5 (PRR9/7/5) recognizing a specific DNA sequence called evening element [27] (Figure $2 A)$. PRRs are repressors that contain in their $\mathrm{N}$-terminus a receiver-like domain (RLD) similar to the one in RESPONSE REGULATORS involved in the His-Asp phosphorelay system, while in the C-terminus they present a CO, COL1 and TIME OF CAB EXPRESSION 1 (TOC1) domain (CCT). In Arabidopsis PRR9, PRR7 and PRR5 present a series of successive expression peaks at early morning, mid-day and afternoon respectively (Figure $2 \mathrm{C}$ ) and their protein products bind to the same positions at the CCA1 promoter where a G-box can be found, repressing its expression during the entire day, and completing the positive/negative feedback morning loop [31].

TOC1 (PRR1) is directly repressed by CCA1 using the evening element located in its promoter. TOC1 in turn also acts as a repressor of CCA1 forming the central negative feedback loop [31]. PRRs homologues have been identified in all plant taxa. A single $P R R$ gene was identified in the $O$. tauri genome (OtTOC1) presenting a similar expression profile as TOC1 and PRR5 (Figure $2 \mathrm{C}$ ) and symmetric to the one in OtCCA1, suggesting that the central loop was already established in algae and is conserved across the entire eukaryotic green lineage [29]. Additionally, an evening element has been found in the promoter of OtTOC1 providing supporting evidence. None of the two PRRs identified in Chlamydomonas follow similar expression profiles to any of the PRRs in Arabidopsis, which suggests a divergent evolution in Chlamydomonas in the regulation of circadian rhythms [32]. Four different PRRs were found in the Physcomitrella (a moss that seems to have derived from the direct evolutionary line of modern plants) genome. All these genes exhibit the same expression pattern, peaking at dusk with their troughs at dawn. No diversification in their peaking time points like the Arabidopsis PRR9/7/5 is observed, possibly because these genes are the result of very recent duplication events [33]. 
The third feedback loop is called the evening loop where TOC1 also plays a key role together with $\mathrm{GI}$ and ZTL (Figure 2). Both genes have been shown to be transcriptionally co-regulated directly by CCA1, PRRs and TOC1 [27,31,34]. In turn, GI and ZTL are known to form a complex involved in blue light and temperature sensing that induces TOC1 degradation by the $26 \mathrm{~S}$ proteasome [35]. It is relevant to note that Arabidopsis ZTL homologue, FKF1, together with GI, mediates CYCLING DOF FACTORs (CDFs) degradation and the subsequent CO activation [36], a connection between the circadian clock and photoperiodic flowering. No potential Gl homologue has been identified in microalgae and Physcomitrella, but it is present in other bryophytes such as Marchantia or Selaginella and the seed plants Picea and Oryza, suggesting that this gene is exclusive of land plants [33]. Gl plays also a crucial role in the temperature compensation of the Arabidopsis circadian clock [37] and the defect in temperature compensation observed in Physcomitrella clock has been ascribed to the absence of GI [33]. ZTL presents three different protein domains: N-terminal LOV involved in blue light sensing, F-box that mediates protein ubiquitination, and multiple C-terminal Kelch domains involved in protein interactions. Similar to GI, ZTL homologs have only been identified in land plants, although the three domains of ZTL have been separately identified in different proteins in Physcomitrella, so that these proteins could form a complex functionally equivalent to ZTL [33]. Alternatively, a closer inspection of the RLD domains in the PRR genes in Physcomitrella and Ostreococcus has revealed a potential phosphoaceptor DDK motif that is not present in the PRRs of land plants. This could indicate that these PRRs form part of a Hist-Asp phosphorelay system. In fact, Histidine kinases containing an N-terminal LOV domain has been identified in Ostreococcus where it has been shown to respond to blue light, bind flavin and have a circadian function [38]. Therefore, these LOV-HKs could possibly take the role of ZTL in Physcomitrella, Chlamydomonas and Ostreococcus.

The evening loop includes also LUX ARRHYTHMO, ELF3 and ELF4 that repress circadian genes night expression [39]. LUX homologs have been found in all plant taxa examined but no functional characterization is described. Potential ELF3/4 orthologues have been identified in Physcomitrella [33] and other land plants [40] but there seem to be no orthologues in Chlamydomonas [41] or Ostreococcus. Therefore, it seems that the central CCA1/LHY and TOC1 loop was established very early in microalgae and the subsequent loops and additional control levels took place during the course of the evolution of land plants. This way, the more complex the clock, the more responses can be extracted from it to control new physiological processes, such as the above mentioned temperature compensation exerted by GI [33]. This could explain how land plants acquired a much more versatile control of the external conditions, enhancing their plasticity and capacity to colonize new aerial niches.

\section{Evolution of the photoperiod pathway}


The long day (LD) A. thaliana plant and its photoperiodic control of flowering time has been the model for day length sensing studies. In Arabidopsis CO protein represents a central hub that controls flowering in the proper season (Figure 3, right). Briefly, CO expression is regulated by circadian clock and photoperiodic inputs through GI-FKF1, the CDFs and FLOWERING BHLHs (FBHs). CO protein stability is controlled by photoreceptors, E3 ubiquitin ligases [42] and through the interaction with other proteins $[43,44]$. These complex regulatory layers allow the correct expression of the florigen FLOWERING LOCUS T (FT) gene that promotes flowering.

However, there are diverse and specific photoperiodic flowering regulatory strategies. In the short day (SD) plant rice, CO homologue functions as an inhibitor in LD and activator in SD [45], while another COL suppresses flowering regardless of day length [46]. In Medicago and pea, CO seems to have no significant effect in flowering time and specifically in the latter, FT expression is controlled solely by a CDF gene $[47,48]$. Nonetheless, evolution goes even further, altering flowering time at the species level by natural variation in the cis-regulatory elements of the CO promoter [49]. Moreover, a local adaptation of ecotypes to different flowering strategies depending on their geographic location has been described [50]. These examples and others [51-54] show how plants have adapted to optimize their reproductive timing.

Although the belief that $\mathrm{CO}$ is a widespread central element in the angiosperms flowering pathway is generating controversy [47,55], the GI-CDFs-CO-FT core is highly conserved among distantly related flowering plants. In fact, it has been shown that COLs and DOFs regulators have coevolved from single common algal ancestors, following the innovation, amplification and divergence model of gene evolution by duplication [56]. C. reinhardtii is considered to be the representative species of this common ancestor, in which, a single copy gene of $\mathrm{CO}(\mathrm{CrCO})$ that controls the expression of a single copy DOF (CrDOF) has been characterized [18,57]. CrCO is a central hub involved in key physiological processes such as carbon metabolism and cell cycle, so that its expression is controlled by photoperiod and its mutation severely effects the capacity of the algae to synthesize starch and to synchronize cell division and growth [18,56]. Furthermore, CrDOF induces, unlike Arabidopsis, CrCO expression in SD by direct binding to its promoter. However, in LD, it represses cell cycle progression in a CrCO-independent way [57] (Figure 3). Surprisingly, both genes phenocopied its homologue functions in Arabidopsis when ectopically expressed; $\mathrm{CrCO}$ inducing flowering time while CrDOF delays it [18,57]. Curiously, COL1 (which shares $80 \%$ amino acid similarity with $\mathrm{CO}$ ) overexpression produces no change in flowering time and this might reveal another evolutionary aspect: It must be the tertiary structure of the algal proteins what is conserved and recognized by the plant regulatory mechanisms to emulate its plant homologue function [56]. This does not seem to be an isolated event because a similar case was observed when tomato CDFs were expressed in Arabidopsis [58]. 
In vascular plants, a great numbers of DOFs and COLs functions have been inherited from their common ancestor. For example, CO triggers starch biosynthesis and the DOF transcription factor OBP1 controls cell cycle $[59,60]$. So, not only DOF-CO module has coevolved, but also a set of genes or key regulatory networks associated to these genes. Additionally, COLs and DOFs have acquired, throughout evolution, a wide repertoire of plant specific light-dependent functions $[5,61,62]$ leading to more complex organism with a higher photoperiod plasticity.

\section{Discussion}

Over evolutionary time, photosynthetic organisms have learnt to live and extract information from periodic changes in sunlight [1,2]. As the complexity of organisms increased, so did their capacity to respond to the environment and paradoxically, to become more independent from its rigours and more precise at taking crucial life decisions, such as the best time of the year to flower or the best time of the day to grow. The massive amount of information arising from comparative genomics projects is allowing us to understand photoperiodic sensing with an evolutionary perspective.

\section{Acknowledgements}

The authors would like to thank funding from projects $\mathrm{BIO} 2011-28847-\mathrm{CO2}-00$ and BIO2014-52425-P (Spanish Ministry of Economy and Competitiveness, MINECO) partially supported by FEDER funding.

\section{Figure Legends}

Figure 1: Light signal transduction and photoperiodic regulation by COP1 in A. thaliana (A) and $C$. reinhardtii (B). In $A$. thaliana, photoactivated CRY1, CRY2, PHYA and PHYB inhibit COP1 allowing accumulation of effectors and resulting in the specific light responses. Under UV-B, COP1 acts as positive regulator activating, among others, UVR8, HY5 and consequently upregulating UV-B tolerance genes. UV-B signal transduction is conserved in C. reinhardtii, although CrHY5 implication has not been investigated (dash lines). Arrows indicate positive regulation, while bars represent negative regulation. Low levels of $\mathrm{CrCO}$ expression observed in crcrya mutant suggest a conserved CRY-COP1-CO pathway, although CrCOP1 implication has not been described (dash lines). Photoreceptors not involved in COP1 regulation are shown in grey.

Figure 2. Evolution of circadian clocks from algae to plants. (A) Transcriptional network underpinning the circadian clock in Arabidopsis thaliana based on occupancy profiling by high throughput sequencing or ChIP-seq of CCA1, TOC1, PRR5, PRR7 and PRR9. Three different loops are identified, the morning loop constituted by CCA1 and PRR9/7/5, the central loop with TOC1 and CCA1 and finally, the evening loop formed by TOC1, GI, ZTL and LUX. (B) Identification of orthologues circadian clock proteins in different plant taxa including gene ID from specific databases. Notice how proteins in the morning and central loops are conserved across the entire green lineage, while the key proteins in the evening loop, ZTL and GI, are only present from Selaginella on. Protein domains (RLD, CCT, Myb, LOV, Fbox and Kelch) are color- 
coded identified on the table on the left. (C) Conservation of the expression profiles of CCA1, PRR $9 / 7 / 5$ and TOC1 from Arabidopsis thaliana (left) and OtCCA1 and OtTOC1 from Ostreococcus tauri (right) in $24 \mathrm{~h}$ experiments at $12 \mathrm{~h}$ light/12h dark photoperiod. CCA1 and OtCCA1 present similar expression profiles peaking at dawn with a trough at dusk. OtTOC1 and TOC1 exhibit similar expression patterns peaking at dusk with a trough at dawn.

Figure 3. Evolution of the photoperiod pathway elements from a common ancestor. In Arabidopsis (right) the main elements of flowering photoperiod pathway are shown. Grey and purple proteins represent phytocromes and E3 ubiquitine ligase proteins, respectively. Straight and dash arrows show transcriptional and post-transcriptional regulation, respectively. Colored circles represent light quality. In Chlamydomonas (left) $\mathrm{CrCO}$ and $\mathrm{CrDOF}$ are the first known elements involved in photoperiodic signaling (there is no evidence of $\mathrm{Gl}$ and FT proteins presence in green algae). Although HOS1, COP1 and FBHs orthologues are present in the Chlamydomonas genome, no functional characterization has been done. The big family of COLs and DOFs in angiosperms has plant specific functions. However, other functions are shared with the Chlamydomonas proteins (dash lines).

\section{References}

1. Lagercrantz U: At the end of the day: a common molecular mechanism for photoperiod responses in plants? J Exp Bot 2009, 60: 2501-2515.

2. Dring, MJ: Photocontrol of development in algae. Ann. Rev. Plant Physiol. Plant Mol. Biol. 1988, 39: 157-174.

3. Monnier A, Liverani S, Bouvet $\mathrm{R}$ et al.: Orchestrated transcription of biological processes in the marine picoeukaryote Ostreococcus exposed to Light/dark Cycles. BMC Genomics 2010, 11: 92.

4. Michael TP, Mockler TC, Breton G et al.: Network discovery pipeline elucidates conserved time-of-day-specific cis-regulatory modules. PloS Genet 2008, 4: e14.

5. Lucas-reina E, Ortiz-Marchena MI, Romero-Campero FJ et al.: Evolution of the flowering pahtways. Prog Bot 2016, 77:291-331.

6. Li FW, Mathews S: Evolutionary aspects of plant photoreceptors. J Plant Res 2016, 129: $115-122$

7. Rizzini L, Favory JJ, Cloix C et al.: Perception of UV-B by the Arabidopsis UVR8 protein. Science 2011, 332: 103-106.

8. Li FW, Melkonian M, Rothfels CJ et al.: Phytochrome diversity in green plants and the origin of canonical plant phytochromes. Nat Commun 2015, 6: 7852.

9. Rensing SA, Sheerin DJ, Hiltbrunner A: Phytochromes: More Than Meets the Eye. Trends Plant Sci 2016, 21: 543-546.

10. Petroutsos D, Tokutsu R, Maruyama S, et al.: A blue-light photoreceptor mediates the feedback regulation of photosynthesis. Nature 2016, 537: 563-566. 
11. Chaves I, Pokorny R, Byrdin $M$ et al.: The cryptochromes: blue light photoreceptors in plants and animals. Annu Rev Plant Biol 2011, 62: 335-364.

12. Kianianmomeni A, Hallmann, A: Algal photoreceptors: in vivo functions and potential applications. Planta 2014, 239: 1-26.

13. Christie JM, Blackwood L, Petersen J, Sullivan S: Plant flavoprotein photoreceptors. Plant Cell Physiol 2014, 56: 401-413.

14. Tilbrook K, Dubois $M$, Crocco $C D$ et al.: UV-B Perception and Acclimation in Chlamydomonas reinhardtii. Plant Cell 2016, 28: 966-983.

15. Beel B, Prager $k$, Spexard $M$ et al.: A flavin binding cryptochrome photoreceptor responds to both blue and red light in Chlamydomonas reinhardtii. Plant Cell 2012, 24: 2992-3008.

16. Kagawa T, Kasahara M, Abe T, Yoshida S, Wada M: Functional analysis of phototropin2 using fern mutants deficient in blue light-induced chloroplast avoidance movement. Plant Cell Physiol 2004, 45: 416-426.

17. Valverde F, Mouradov A, Soppe $W$ et al.: Photoreceptor regulation of CONSTANS protein in photoperiodic flowering. Science 2004, 303: 1003-1006.

18. Serrano G, Herrera-Palau R, Romero JM et al.: Chlamydomonas CONSTANS and the evolution of plant photoperiodic signaling. Curr Biol 2009, 19: 359-368.

19. Huang $\mathrm{H}$, Yoo $\mathrm{CY}$, Bindbeutel $\mathrm{R}$ et al.: $\mathrm{PCH} 1$ integrates circadian and light-signaling pathways to control photoperiod-responsive growth in Arabidopsis. Elife 2016, 5: e13292.

20. Lau OS, Deng XW: The photomorphogenic repressors COP1 and DET1: 20 years later. Trends Plant Sci 2012, 17: 584-593.

21. Jang S, Marchal V, Panihagri KCS et al.: Arabidopsis COP1 shapes the temporal pattern of CO accumulation conferring a photoperiodic flowering response. EMBO J 2012, 27: 12771288.

22. Lu XD, Zhou $C M, X u$ PB et al.: Red-light-dependent interaction of phyB with SPA1 promotes COP1-SPA1 dissociation and photomorphogenic development in Arabidopsis. Mol Plant 2015, 8: 467-478.

23. Yu JW, Rubio V, Lee NY et al.: COP1 and ELF3 control circadian function and photoperiodic flowering by regulating GI stability. Mol Cell 2008, 32: 617-630.

24. Yin R, Skvortsova MY, Loubéry S, Ulm R: COP1 is required for UV-B-induced nuclear accumulation of the UVR8 photoreceptor. Proc Natl Acad Sci U S A 2016, 113: 4415-4422.

25. Nohales MA, Kay SA: Molecular mechanisms at the core of the plant circadian oscillator. Nat Struct Mol Biol 2016, 23: 1061-1069.

26. McClung CR: Wheels within wheels: new transcriptional feedback loops in the Arabidopsis circadian clock. F1000Prime Rep 2014, 6: 2.

27. Kamioka M, Takao S, Suzuki T et al.: Direct repression of evening genes by CIRCADIAN CLOCK-ASSOCIATED 1 in the Arabidopsis circadian clock. Plant Cell 2016, 28: 696-711.

28. Du H, Wang YB, Xie $Y$ et al.: Genome-wide identification and evolutionary and expression analyses of MYB-related genes in land plants. DNA Res 2013, 20: 437-448. 
29. Corellou F, Schwartz C, Motta JP et al.: Clocks in the green lineage: comparative functional analysis of the circadian architecture of the picoeukaryote Ostreococcus. Plant Cell 2009, 21 : 3436-3449.

30. Hsu PY, Devisetty UK, Harmer SL: Accurate timekeeping is controlled by a cycling activator in Arabidopsis. Elife 2013, 2: e00473.

31. Liu TL, Newton L, Liu MJ et al.: A G-Box-like motif is necessary for transcriptional regulation by circadian Pseudo-Response Regulators in Arabidopsis. Plant Physiol 2016, 170: 528-539.

32. Mittag $\mathrm{M}$, Kiaulehn $\mathrm{S}$, Johnson $\mathrm{CH}$ : The circadian clock in Chlamydomonas reinhardtii. What is it for? What is it similar to? Plant Physiol 2015, 137: 399-409.

33. Holm K, Källman T, Gyllenstrand $\mathrm{N}$ et al.: Does the core circadian clock in the moss Physcomitrella patens (Bryophyta) comprise a single loop? BMC Plant Biol 2010, 10: 109.

34. Huang W, Pérez-García P, Pokhilko A et al.: Mapping the core of the Arabidopsis circadian clock defines the network structure of the oscillator. Science 2012, 336: 75-79.

35. Miyazaki $\mathrm{Y}$, Takase $\mathrm{T}$, Kiyosue T: ZEITLUPE positively regulates hypocotyl elongation at warm temperature under light in Arabidopsis thaliana. Plant Signal Behav 2015, 10: e998540.

36. Song YH, Estrada DA, Johnson RS et al.: Distinct roles of FKF1, GIGANTEA, and ZEITLUPE proteins in the regulation of CONSTANS stability in Arabidopsis photoperiodic flowering. Proc Natl Acad Sci USA 2014, 111: 17672-17677.

37. Gould PD, Locke JC, Laure $\mathrm{C}$ et al.: The molecular basis of temperature compensation in the Arabidopsis clock. Plant Cell 2006, 18: 1177-1187.

38. Djouani-Tahri E, Christie JM, Sanchez-Ferandin S et al.: A eukaryotic LOV-histidine kinase with circadian clock function in the picoalga Ostreococcus. Plant J 2011, 65: 578-588.

39. Mizuno T, Kitayama M, Takayama C, Yamashino T: Insight into a Physiological Role for the EC Night-Time Repressor in the Arabidopsis Circadian Clock. Plant Cell Physiol 2015, 56: 17381747.

40. Murakami M, Tago Y, Yamashino T, Mizuno T: Comparative overviews of clock-associated genes of Arabidopsis thaliana and Oryza sativa. Plant Cell Physiol 2007, 48: 110-121.

41. Ryo M, Matsuo T, Yamashino $T$ et al.: Diversity of plant circadian clocks: Insights from studies of Chlamydomonas reinhardtii and Physcomitrella patens. Plant Signal Behav 2016, 11: e1116661.

42. Shim JS, Kubota A, Imaizumi T: Circadian clock and photoperiodic flowering in Arabidopsis: CONSTANS is a hub for signal integration. Plant Physiol 2016, doi:10.1104/pp.16.01327.

43. Wang C, Guthrie C, Sarmast MK, Dehesh K: BBX19 Interacts with CONSTANS to Repress FLOWERING LOCUS T Transcription, Defining a Flowering Time Checkpoint in Arabidopsis. Plant Cell 2014, 24:3589-3602.

44. Graeff M, Straub D, Eguen T, Dolde U: MicroProtein-mediated recruitment of CONSTANS into a TOPLESS trimeric complex represses flowering in Arabidopsis. PLoS Genet 2016, 12:122. 
45. Yano $M$, Katayose $\mathrm{Y}$, Ashikari $\mathrm{M}$ et al.: Hd1, a major photoperiod sensitivity quantitative trait locus in rice, is closely related to the Arabidopsis flowering time gene CONSTANS. Plant Cell 2000, 12:2473-2484.

46. Sheng $\mathrm{P}, \mathrm{Wu} \mathrm{F}$, Tan J, Zhang $\mathrm{H}, \mathrm{Ma} \mathrm{W}$ : A CONSTANS-like transcriptional activator, OsCOL13, functions as a negative regulator of flowering downstream of OsphyB and upstream of Ehd1 in rice. Plant Mo. Bio. 2016, doi:10.1007/s11103-016-0506-3.

47. Ridge $S$, Sussmilch FC, Hecht $V$ et al.: Identification of LATE BLOOMER2 as a CYCLING DOF FACTOR Homolog Reveals Conserved and Divergent Features of the Flowering Response to Photoperiod in Pea. Plant Cell 2016, 28:2545-2559.

48. Wong $\mathrm{A}$, Hecht $\mathrm{V}$, Picard $\mathrm{K}$ et al.: Isolation and functional analysis of CONSTANS-LIKE genes suggests that a central role for CONSTANS in flowering time control is not evolutionarily conserved in Medicago truncatula. Front Plant Sci 2014, 1:1-10.

49. Rosas U, Mei Y, Xie Q et al.: Variation in Arabidopsis flowering time associated with cis regulatory variation in CONSTANS. Nat Commun 2014, 5:1-8.

50. Vanhala T, Normann KR, Lundström M et al.: Flowering time adaption in Swedish landrace pea (Pisum sativum L.). BMC Genet 2016, doi:10.1186/s12863-016-0424-z.

51. Li D, Yang C, Li X et al.: Functional characterization of rice OsDof12. Planta 2009, 229:1159-69.

52. Lee Y, An G: OsGI Controls Flowering Time by Modulating Rhythmic Flowering Time Regulators Preferentially under Short Day in Rice. J Plant Biol 2015, 1:137-145.

53. Kloosterman B, Abelenda JA, Gomez MDMC et al.: Naturally occurring allele diversity allows potato cultivation in northern latitudes. Nature 2013, 495:246-250.

54. Cao D, Li Y, Lu S et al.: GmCOL1a and GmCOL1b Function as Flowering Repressors in Soybean Under Long-Day Conditions. Plant Cell Physiol 2015, 56:2409-2422.

55. Simon S, Rïl M, Montaigu A De, Wötzel S, Coupland G: Evolution of CONSTANS Regulation and Function after Gene Duplication produced a Photoperiodic Flowering Switch in the Brassicaceae. Mol Biol Evol 2015, 32:2284-2301.

56. Romero-Campero FJ, Lucas-Reina E, Said FE, Romero JM, Valverde F: A contribution to the study of plant development evolution based on gene co-expression networks. Front Plant Sci 2013, 4:1-17.

57. Lucas-Reina E, Romero-Campero FJ, Romero JM, Valverde F: An Evolutionarily Conserved DOF-CONSTANS Module Controls Plant Photoperiodic Signalling. Plant Physiol 2015, 168:561-574.

58. Corrales A-R, Nebauer SG, Carrillo L et al.: Characterization of tomato Cycling Dof Factors reveals conserved and new functions in the control of flowering time and abiotic stress responses. J Exp Bot 2014, 65:995-1012.

59. Ortiz-Marchena MI, Albi T, Lucas-Reina E et al.: Photoperiodic Control of Carbon Distribution during the Floral Transition in Arabidopsis. Plant Cell 2014, 26:565-584.

60. Skirycz A, Radziejwoski A, Busch W et al.: The DOF transcription factor OBP1 is involved in cell cycle regulation in Arabidopsis thaliana. Plant J 2008, 56:779-92. 
61. Valverde F: CONSTANS and the evolutionary origin of photoperiodic timing of flowering. $J$ Exp Bot 2011, 62:2453-63.

62. Noguero M, Atif RM, Ochatt S, Thompson RD: The role of the DNA-binding One Zinc Finger (DOF) transcription factor family in plants. Plant Sci 2013, 209:32-45.

\section{Commented references}

- 14. Tilbrook K, Dubois M, Crocco CD et al.: UV-B Perception and Acclimation in Chlamydomonas reinhardtii. Plant Cell 2016, 28: 966-983.

In this paper, a clear demonstration of evolutionary conserved UVR8 UV-B perception and signalling from $C$. reinhardtii to $A$. thaliana is shown.

- 18. Serrano G, Herrera-Palau R, Romero JM et al:: Chlamydomonas CONSTANS and the evolution of plant photoperiodic signaling. Curr Biol 2009, 19: 359-368.

Authors demonstrate that there is a conserved role of $\mathrm{CO}$ in photoperiodic signalling from $C$. reinhardtii to $A$. thaliana and that it was involved in cell cycle progression and starch synthesis in algae.

- 27. Kamioka M, Takao S, Suzuki T et al.: Direct repression of evening genes by CIRCADIAN CLOCK-ASSOCIATED 1 in the Arabidopsis circadian clock. Plant Cell 2016, 28: 696-711.

In this paper high throughput sequencing (ChIP-seq) were performed in order to determine CCA1 regulome and elucidate part of the transcriptional network corresponding to the morning loop, finding that CCA1 binding sites are significantly enriched in evening elements and G-boxes.

- 29. Corellou F, Schwartz C, Motta JP et al.: Clocks in the green lineage: comparative functional analysis of the circadian architecture of the picoeukaryote Ostreococcus. Plant Cell 2009, 21: 3436-3449.

In this paper OtCCA1 and OtTOC1 were identified and functionally characterized in Ostreococcus tauri, supporting a common evolutionary origin of circadian clock in the green lineage that is already established in Chlorophyta.

- 34. Huang W, Pérez-García P, Pokhilko A et al.: Mapping the core of the Arabidopsis circadian clock defines the network structure of the oscillator. Science 2012, 336: 75-79.

In this paper the TOC1 regulome was described, elucidating part of the transcriptional network corresponding to the evening loop. They showed that TOC1 acts as a repressor.

- 49. Rosas U, Mei Y, Xie Q et al.: Variation in Arabidopsis flowering time associated with cis regulatory variation in CONSTANS. Nat Commun 2014, 5:1-8.

Demonstration of the recent origin of $\mathrm{CO}$ promoter polymorphism and how its natural variation regulates flowering time in different Arabidopsis thaliana accessions.

- 55. Simon S, Rïl M, Montaigu A De, Wötzel S, Coupland G: Evolution of CONSTANS Regulation and Function after Gene Duplication produced a Photoperiodic Flowering Switch in the Brassicaceae. Mol Biol Evol 2015, 32:2284-2301.

An elegant demonstration of how the duplication of a core photoperiodic gene has a deep effect on the adaptation and flowering behaviour of a whole family of plants and the possible convergence of this function in different plant lineages. 
- 57. Lucas-Reina E, Romero-Campero FJ, Romero JM, Valverde F: An Evolutionarily Conserved DOF-CONSTANS Module Controls Plant Photoperiodic Signalling. Plant Physiol 2015, 168:561-574.

By functional characterization of CrDOF in algae and plants, CO-DOF module is shown to be conserved throughout the green linage evolution. 


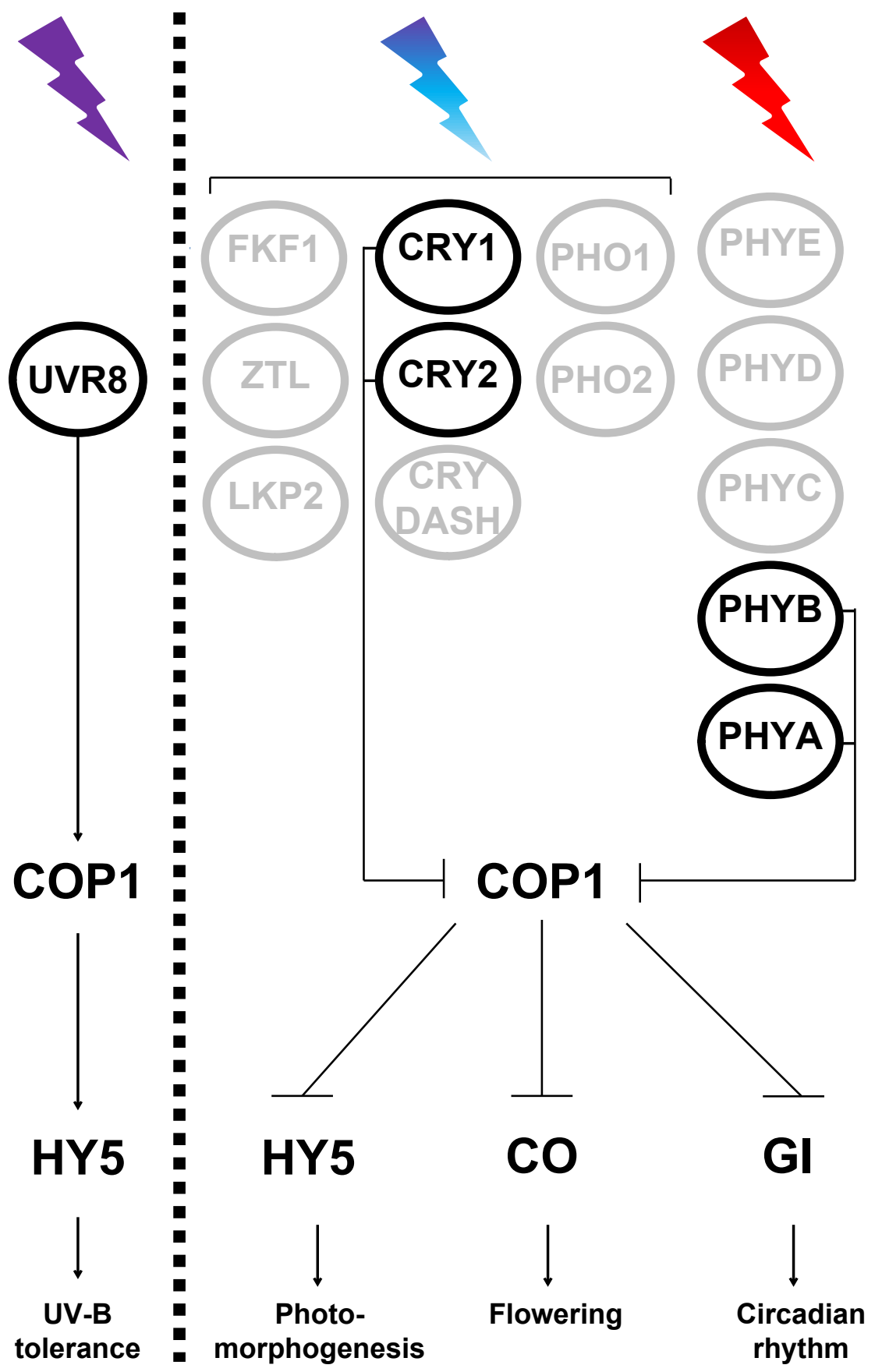

(B)

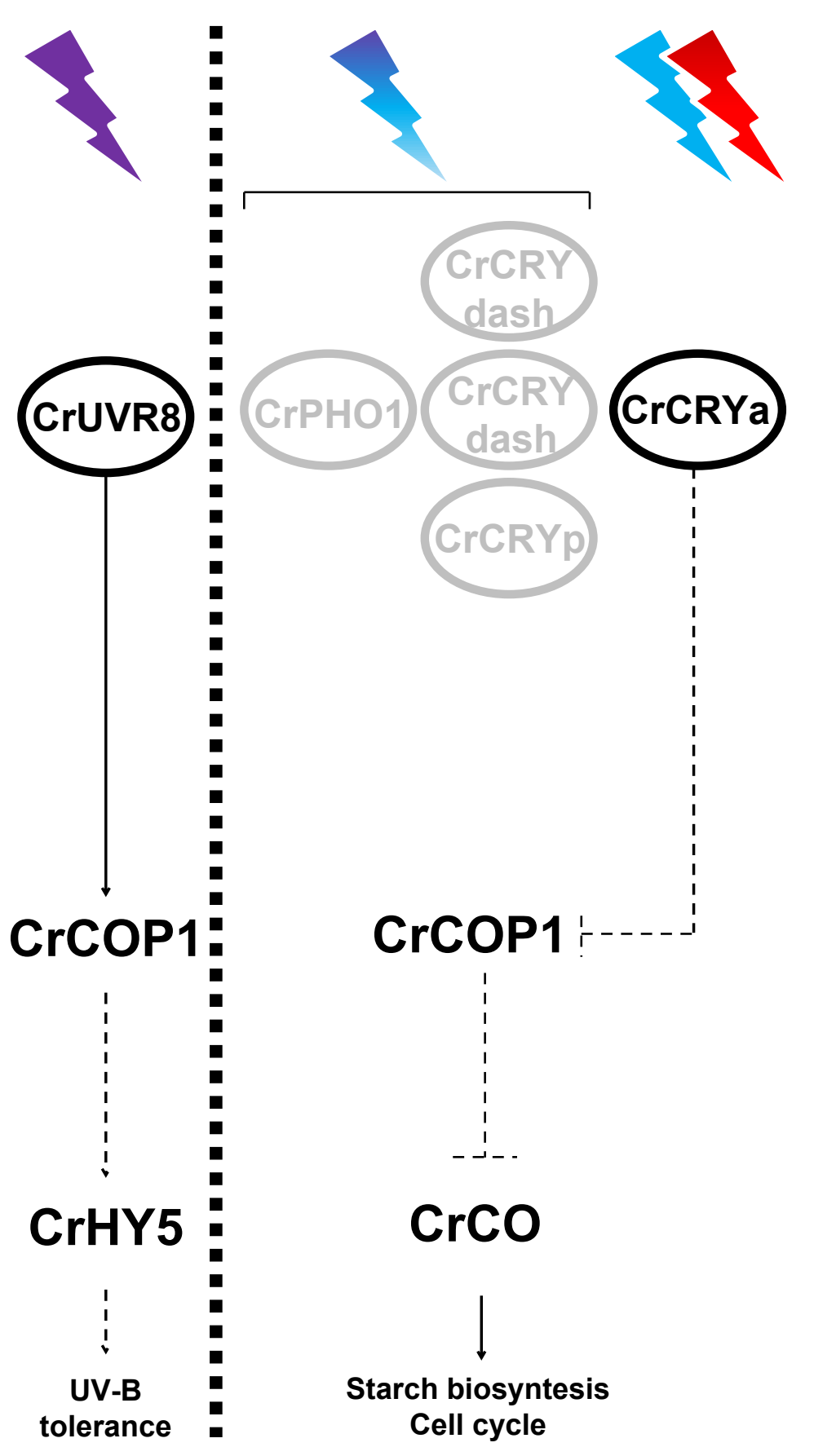


(B)

\section{Arabidopsis thaliana}

(A) Morning Loop Central Loop
Evening Loop

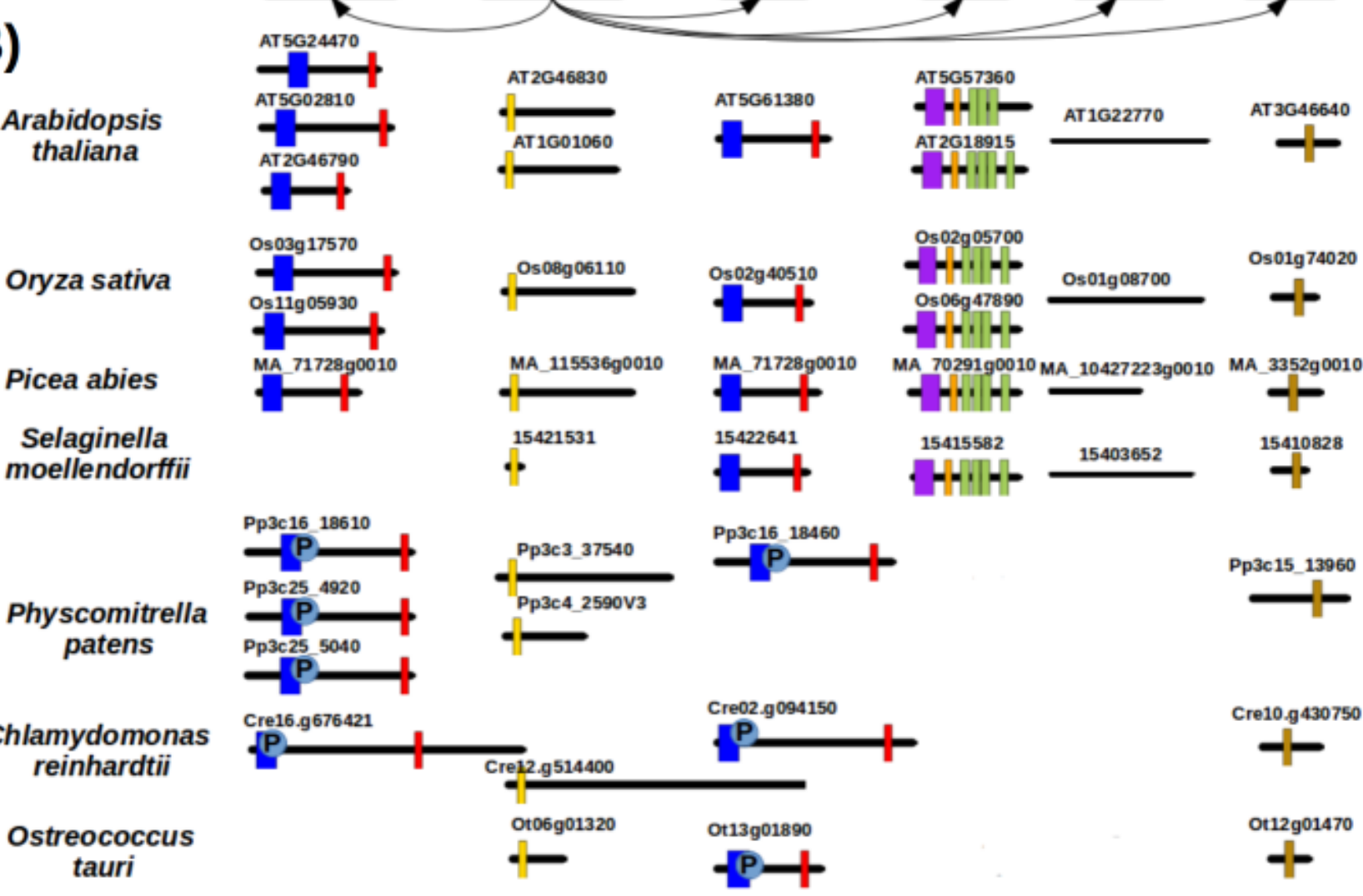




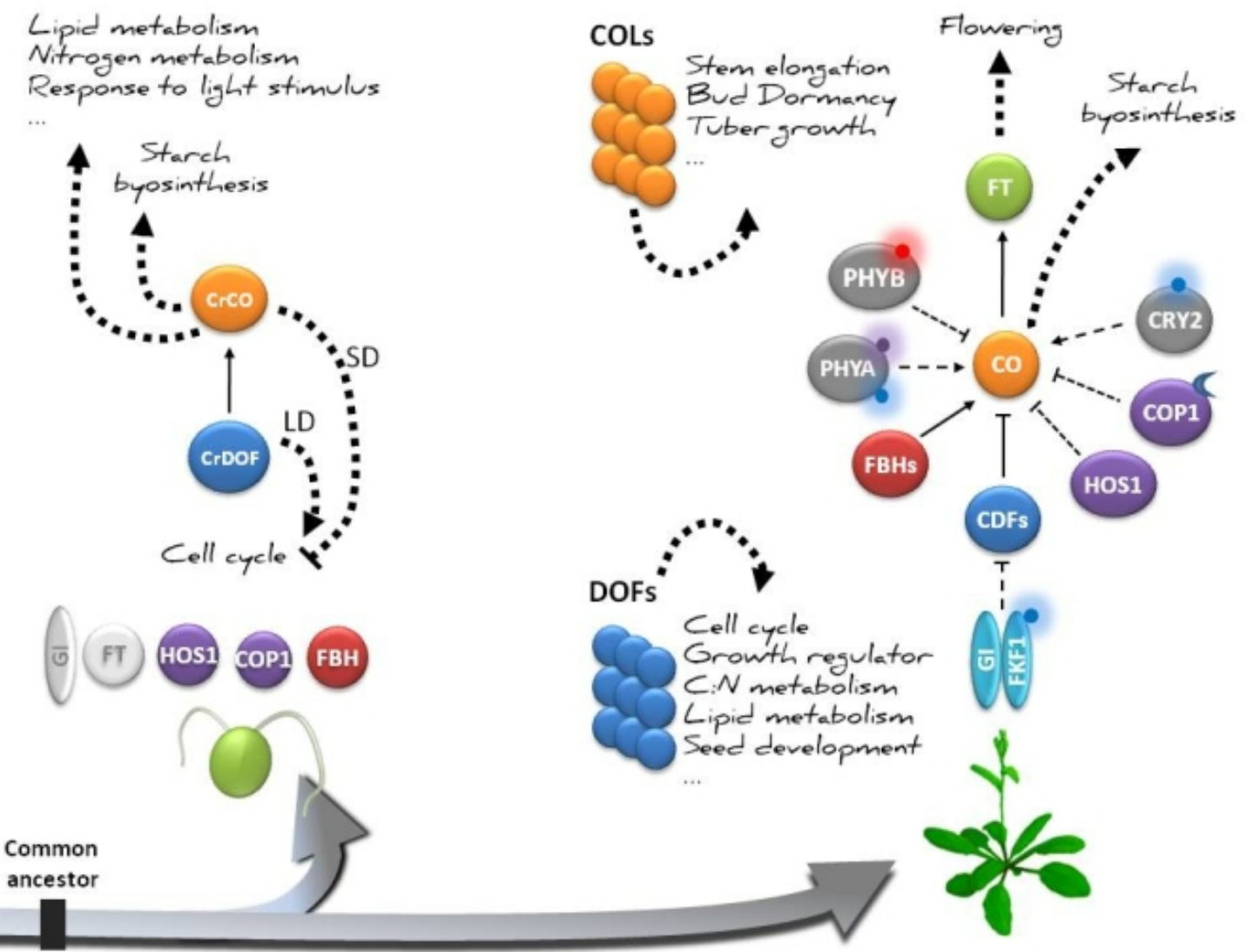




\section{Highlights}

- The mechanisms to detect day length are present in almost all eukaryotic photosynthetic organisms known to date.

- The photoperiodic gene toolkit present in modern plants first appeared in microalgae.

- COP1-like proteins seem to play a central role during plant photoperiodic sensing evolution.

- The circadian clock core appeared in algae and developed complex secondary loops during plant evolution. 